\title{
A Mathematical Simulation of the Ureter: Effects of the Model Parameters on Ureteral Pressure/Flow Relations
}

Ureteral peristaltic mechanism facilitates urine transport from the kidney to the bladder. Numerical analysis of the peristaltic flow in the ureter aims to further our understanding of the reflux phenomenon and other ureteral abnormalities. Fluid-structure interaction (FSI) plays an important role in accuracy of this approach and the arbitrary Lagrangian-Eulerian (ALE) formulation is a strong method to analyze the coupled fluidstructure interaction between the compliant wall and the surrounding fluid. This formulation, however, was not used in previous studies of peristalsis in living organisms. In the present investigation, a numerical simulation is introduced and solved through ALE formulation to perform the ureteral flow and stress analysis. The incompressible NavierStokes equations are used as the governing equations for the fluid, and a linear elastic model is utilized for the compliant wall. The wall stimulation is modeled by nonlinear contact analysis using a rigid contact surface since an appropriate model for simulation of ureteral peristalsis needs to contain cell-to-cell wall stimulation. In contrast to previous studies, the wall displacements are not predetermined in the presented model of this finite-length compliant tube, neither the peristalsis needs to be periodic. Moreover, the temporal changes of ureteral wall intraluminal shear stress during peristalsis are included in our study. Iterative computing of two-way coupling is used to solve the governing equations. Two phases of nonperistaltic and peristaltic transport of urine in the ureter are discussed. Results are obtained following an analysis of the effects of the ureteral wall compliance, the pressure difference between the ureteral inlet and outlet, the maximum height of the contraction wave, the contraction wave velocity, and the number of contraction waves on the ureteral outlet flow. The results indicate that the proximal part of the ureter is prone to a higher shear stress during peristalsis compared with its middle and distal parts. It is also shown that the peristalsis is more efficient as the maximum height of the contraction wave increases. Finally, it is concluded that improper function of ureteropelvic junction results in the passage of part of urine back flow even in the case of slow start-up of the peristaltic contraction wave. [DOI: $10.1115 / 1.4003316]$

Keywords: peristalsis, vesicoureteral reflux, arbitrary Lagrangian-Eulerian formulation, FSI method

\section{Introduction}

Peristaltic motion consists of successive sequence of contractions along a muscular tube. This motion is the driving force behind many critical functions in the human body such as transportation of urine from the kidney to the bladder. The mechanism that governs the ureteral peristalsis has been a focal point since the early beginning of peristaltic research [1]. Despite extensive research [1-3], this mechanism has not yet been fully understood, neither the pertaining mechanical factors have been analyzed precisely. For example the effects of ureteral wall compliance and inlet/outlet pressure differences on the peristaltic efficiency have not been accurately explained yet.

Stimulation of the ureter in peristalsis creates a contraction wave that continuously travels through a cell-to-cell propagation mechanism from the site of stimulation to the bladder [4]. The passage of urine in the ureter cannot be completely defined by peristaltic contractions. Indeed, it is known that the pressure dif-

Contributed by the Bioengineering Division of ASME for publication in the JouRNAL OF BIOMECHANICAL ENGINEERING. Manuscript received April 23, 2010; final manuscript received December 13, 2010; accepted manuscript posted December 22, 2010; published online February 4, 2011. Assoc. Editor: Dalin Tang. ference between the renal pelvis and the bladder plays an observable role in the ureteral flow [3]. In a healthy ureter, ureteral peristalsis occurs only $1-5$ times $/ \mathrm{min}$. When there is no peristaltic contraction (at the time intervals between two pace maker activations), the ureter can be seen as a passive tube that yields a steady flow of urine [2].

The irregular flow of urine from the bladder into the ureter, and possibly on to the kidneys, is known as vesicoureteral reflux. In severe cases, vesicoureteral reflux may allow toxins and bacteria from the bladder to infect and hamper kidney function [3], thus leading to dialysis or in the critical cases, kidney transplantation [5].

A quantitative analysis to characterize urine flow will further aid our understanding of the ureter and also aid in the design of flow aided devices such as valves and stents to correct reflux conditions. The quantitative studies carried out in the field of experimental biomedicine up to now have been limited to (1) urine volume transported, (2) peristaltic wave propagation rate [6], and (3) morphometry of the ureter [7]. The qualitative studies in the biomedicine have also been focused on (1) variations of ureteral lumen during peristalsis [8], (2) reaction of the ureteral muscular tissue to drug and neurological stimulation [9-13], and (3) differ- 


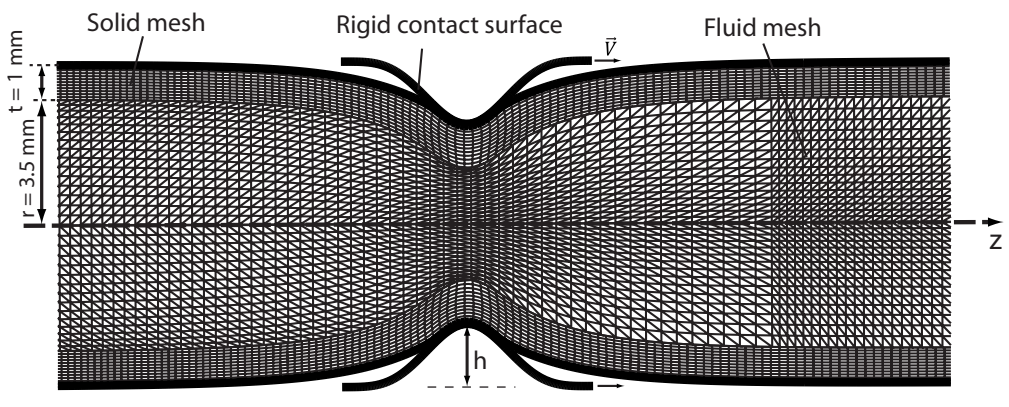

Fig. 1 FSI computational model. As it is shown, the rigid contact surface motion leads to contraction wave propagation in the ureter. $h$ represents the maximum height of contraction wave. The model is axisymmetric although the complete model is presented here for better illustration.

ent flow patterns occurring in the ureter [14-16] of various mammalians. Studies in the field of biomechanics have been concentrated on understanding the mechanical properties of the ureteral wall [17-20] and mathematical analyses of peristaltic flow to be applied to the gastro-intestinal system [21], the urinary system [3,22-25], and other living organs [26,27].

Due to the complexity of urine transport in the ureter, the recent mathematical simulations of the ureteral flow have also been restricted to very simple (two-dimensional, 2D) geometries and boundary conditions [28-31]. The physiological function pertaining to the contraction rate of smooth muscle in the ureter is extremely complicated, and therefore, the ureteral smooth muscle has not yet been accurately modeled [32]. Three factors are identified as influencing the contraction rate of the ureteral smooth muscle: (1) the load against which the muscle is contracting (which largely consists of hydrodynamic (viscous) forces required to move urine), (2) the current ureteral geometry, and (3) its state of activation.

Griffiths [33] published a theoretical analysis with numerical solutions evaluating the peristaltic flow through a distensible tube of limited length. The results showed that for flow with isolated boluses, the pressure/flow relation was determined by the active and passive properties of the tube undergoing peristalsis and not by the outlet load condition. The dynamics of the upper urinary tract and the effect of various bladder pressures on ureteral pressure/flow relations have also been studied by other researchers $[2,15,16,24,28]$ but none of those studies included fluid-structure interaction (FSI) in their analysis. Ureteral wall mechanical properties should not be over sighted in numerical analysis of ureteral function since they determine the significant deformations of ureteral wall, which play an important role in the urine transport from the kidney into the bladder. Wall deformations are caused by the wall stimulation as well as fluid forces, and therefore needed to be studied through fluid-structure interaction. We should mention that in order to reach a reliable simulation, wall configuration should not be predetermined in the ureter during peristalsis because of the associated effects of wall compliance and fluid intraluminal pressure. To the best of our knowledge, this issue was not addressed in previously presented models of finite-length ureter.

We previously modeled a two-dimensional ureteral peristaltic flow [31] that assumed a solid wall moving along the longitudinal direction and between two rigid plates. The foremost shortcoming of that study was that the cell-to-cell stimulation of ureteral wall during peristalsis, which occurs in the physiological condition of ureteral peristalsis, was not modeled. Here, an axisymmetric nonlinear FSI model is presented using real ureteral data. We aim to tackle the problem of ureteral peristaltic activity, taking into account ureteral wall compliance and its cell-to-cell stimulation, in order to investigate the effects of different mechanical parameters on the ureteral flow and stress analysis that results in the better understanding of ureteral function. The main intents of this re- search are (1) analyzing urine flow field in the ureter in order to better understand the reflux phenomenon, (2) quantitative analysis of peristaltic efficiency in different ureteral functional situations, and (3) exploring the temporal changes in ureteral wall shear stress during the peristaltic wave propagation.

\section{Materials and Methods}

The goal of this study is to improve the realism of computational solution of the problem of urine transport from the kidney to the bladder. Numerical simulation with fluid-structure interaction is presented. The computational analysis of a FSI problem requires two modeling steps: the creation of a mathematicalmechanical model and solving the coupled equations of the fluid and the structure.

2.1 Geometry of the Model. The ureter was assumed to be like an axisymmetric tube. The ureteral diameter, including the muscular coat, varies from $4 \mathrm{~mm}$ to $10 \mathrm{~mm}$ [28]. In this study, it was assumed to be $9 \mathrm{~mm}$. Figure 1 illustrates the geometry of the ureteral computational model. The rigid contact surface shown in this figure simulates a deformable wall stimulator that propagates the peristaltic wave through the ureter.

\subsection{Governing Equations}

2.2.1 Fluid. A transient viscous flow in an axisymmetric tube (Fig. 1) was used to obtain desirable equation parameters. The incompressible Navier-Stokes equations were used as the governing equations with the assumptions of laminar and Newtonian flow. The continuity and momentum equations for the incompressible fluid, respectively, are

$$
\begin{gathered}
\nabla \cdot \boldsymbol{u}=\mathbf{0} \\
\rho^{f} \frac{\partial u_{i}}{\partial t}+\rho^{f}\left(u_{j}-\frac{\partial d_{j}^{f}}{\partial t}\right) \frac{\partial u_{i}}{\partial x_{j}}=\frac{\partial \tau_{i j}^{f}}{\partial x_{j}}
\end{gathered}
$$

where $\boldsymbol{u}$ and $u_{i}$ are the fluid velocity vector and the fluid velocity in the direction $i$, respectively, $\rho^{f}$ is the fluid density, and $d_{j}^{f}$ is the fluid displacement along the fluid-structure interface or other moving boundaries. The stress tensor can also be presented as shown below

$$
\tau_{i j}^{f}=-p \delta_{i j}+2 \mu e_{i j}
$$

where $p$ is the fluid pressure, $\delta_{i j}$ is the Kronecker delta, and $\mu$ is the fluid viscosity. Fluid weight term was ignored in the equations. The strain tensor $e_{i j}$ is defined as follows:

$$
e_{i j}=\frac{1}{2}\left(\frac{\partial u_{i}}{\partial x_{j}}+\frac{\partial u_{j}}{\partial x_{i}}\right)
$$

where $u_{i}$ and $u_{j}$ are the fluid velocities in the directions $i$ and $j$, respectively. 
Table 1 Effect of pressure difference between the ureteral inlet and outlet on the urine flow rate at the ureteral outlet in the models with different maximum heights of contraction waves. In these cases, the ureteral Young's modulus and the contraction wave velocity were assumed to be $5 \mathrm{kPa}$ and $2 \mathrm{~cm} / \mathrm{s}$, respectively.

\begin{tabular}{|c|c|c|c|c|}
\hline $\begin{array}{c}\text { Maximum height } \\
\text { of contraction wave }(\mathrm{mm})\end{array}$ & $\begin{array}{l}\text { Ureteral outlet } \\
\text { pressure }(\mathrm{Pa})\end{array}$ & $\begin{array}{l}\text { Ureteral inlet } \\
\text { pressure }(\mathrm{Pa})\end{array}$ & $\begin{array}{l}\text { Ureteral pressure difference } \\
\text { between its outlet and inlet } \\
\qquad(\mathrm{Pa})\end{array}$ & $\begin{array}{c}\text { Flow rate difference } \\
\text { between } \mathrm{t}=12 \mathrm{~s} \text { (peristaltic wave } \\
\text { starting time) and the ending time } \\
\text { of wall peristaltic motion } \\
\text { at the ureteral outlet } \\
\left(\mathrm{cm}^{3} / \mathrm{s}\right)\end{array}$ \\
\hline 1.4 & 129.7 & 130 & +0.3 & $9.4183 \times 10^{-2}$ \\
\hline 1.68 & 129.7 & 130 & +0.3 & $1.4204 \times 10^{-1}$ \\
\hline 1.4 & 130 & 130 & 0 & $1.3831 \times 10^{-1}$ \\
\hline 1.68 & 130 & 130 & 0 & $1.9745 \times 10^{-1}$ \\
\hline 1.4 & 130 & 129.95 & -0.05 & $1.4523 \times 10^{-1}$ \\
\hline 1.68 & 130 & 129.95 & -0.05 & $2.0987 \times 10^{-1}$ \\
\hline
\end{tabular}

2.2.2 Solid. The ureteral wall is modeled mathematically utilizing the classical Lagrangian formulation, which is as follows:

$$
\frac{\partial \tau_{i j}^{s}}{\partial x_{j}}=\rho^{s} \frac{\partial^{2} d_{i}^{s}}{\partial t^{2}}
$$

where $\tau_{i j}^{s}$ is the Cauchy stress tensor, $d_{i}^{s}$ is the solid displacement component, and $\rho^{s}$ is the solid density.

2.2.3 Fluid-Structure Interaction. In this formulation, the coupling state of the fluid and the structure interaction must be satisfied. The kinematic coupling conditions, which represent the noslip conditions at the interface, are

$$
\begin{gathered}
d_{i}^{f}=d_{i}^{s} \\
\frac{\partial d_{i}^{f}}{\partial t}=\frac{\partial d_{i}^{s}}{\partial t}
\end{gathered}
$$

The above equations require that the values of displacement and velocity for both the fluid and the solid be the same at the interface boundary.

The dynamic coupling condition shows the equilibrium of forces as given below

$$
n_{j} \tau_{i j}^{f}=n_{j} \tau_{i j}^{s}
$$

where $n$ is the unit vector normal to the interface of the fluid and solid regions. Equation (7) provides the balance of forces between the fluid and the structure at the interface boundary.

\subsection{Materials Properties and Boundary Conditions}

2.3.1 Fluid. The urine in the ureter was assumed to be homogenous with a constant density of $\rho=1050 \mathrm{~kg} / \mathrm{m}^{3}$ and viscosity of $\mu=1.3 \mathrm{cP}$. The boundary conditions for the fluid regions are (1) no slipping takes place between the fluid and the ureteral wall and that no penetration of the fluid through the ureteral wall occurs; (2) a pressure difference between the outlet and the inlet of the ureter is needed to perform the flow in the nonperistaltic transport of urine. This pressure difference varies between $-0.05 \mathrm{~Pa}$ and $0.3 \mathrm{~Pa}$ in different numerical models (the pressure magnitudes applied to the ureteral inlet and outlet can be seen in Table 1), and (3) FSI conditions are defined between the ureteral wall and the urine on their interface all through the model.

2.3.2 Solid. Material properties for the wall were assumed to be linear elastic, isotropic, incompressible, and homogeneous with two different Young's modulus of E=5 $\mathrm{kPa}$ and $10 \mathrm{kPa}$ that were approximated from the stress/stretch relations reported by Yin and Fung [20] from their experimental studies on the ureteral tissue. The boundary conditions for the wall were (1) the wall was fixed at the inlet and the outlet in the longitudinal direction to prevent rigid body motion error in the finite element model. (2) It was assumed that a rigid contact surface mechanically stimulates a deformable wall (Fig. 1). When the solution reaches the steady state with the correct velocity profile and the right pressure drop along the ureter $(t=12 \mathrm{~s})$, the rigid contact surface moves in the axial direction along the ureter with a constant velocity $(1 \mathrm{~cm} / \mathrm{s}$ to $2 \mathrm{~cm} / \mathrm{s}$ ) similar to that which exits in the physiological ureteral peristalsis $[22,28]$ to model the ureteral peristaltic flow. (3) As for the fluid model, FSI conditions were defined between the ureteral wall and the urine.

2.4 Simulation Process. Discretization changes the form of the partial differential equations to algebraic equations, and therefore facilitates the numerical solution of the equations. Computational fluid dynamics (CFD) uses numerical methods and algorithms to solve fluid flow problems using the discretized form of Newton's second law. For various coupled problems, the fluid traction affects the solid's deformations, while the structural displacement affects the flow field. This verity is the reason to accomplish fluid-structure interaction analyses. In this study, ADINA software (v8.5, ADINA R\&D, Inc., Watertown, MA), which uses the Lagrangian formulation in the structural model and the arbitrary Lagrangian-Eulerian (ALE) formulation in the fluid counterpart, was employed to solve the fully coupled system. The governing equations of the fluid were solved utilizing upwind scheme, finite element methods, and sparse solver (which employs the standard Galerkin method for incompressible fluid flow). The Euler and implicit-Newmark integration methods were used for the fluid and the solid, respectively. An iterative Newtonian method was used for solving the obtained weak form of the governing equations of the fluid and the structure. The number of iterations and iteration tolerance of velocity for the solid equations were set to 300 and 0.01 , respectively. As for the fluid equations, these parameters were assumed to be 500 and 0.001 , respectively, for a reliable convergence. Using direct computing of two-way coupling, the solution obtained was fully coupled and the kinematical conditions of displacement, velocity, and acceleration continuity across the no-slip fluid-solid (ureter-urine) interface were satisfied at all the time steps during the analysis.

2.5 Computational Grid Generation. Fluid domain was meshed with 54990 2D fluid-axisymmetric-type elements. All fluid elements were triangular and contained three nodes. 12960 2D solid-axisymmetric-type elements were used for the solid region. The whole solid elements were quadrilateral and contained nine nodes. The quantity of the elements (referred above) for the fluid and solid models were achieved by gradually increasing the number of elements until the solution became independent from the grid size. The typical refined mesh is shown in Fig. 1. The maximum solution time for the models was about $22 \mathrm{~h}$ on a 1.8 $\mathrm{GHz}$ Xeon dual core Intel (0.768 GB random access memory (RAM)) processor. 


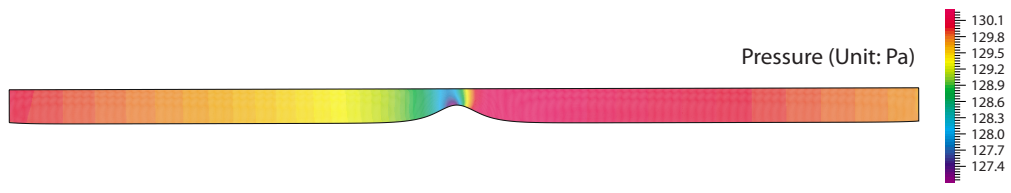

Fig. 2 Ureteral pressure profile during peristaltic wave propagation. The results are related to the case of $E=5 \mathrm{kPa}, V=2 \mathrm{~cm} / \mathrm{s}, \mathrm{h}=1.68 \mathrm{~mm}$, and $\Delta p=0.3 \mathrm{~Pa}$. The pressure profile is relatively similar around the contraction wave peak during all the solution time.

\section{Results}

In this paper, a more proper mechanical model was presented compared with previous studies. The main benefit of this model is that multifunctional factors that are essential in the ureteral performance were considered together. Moreover, ureteral muscular contraction was modeled using an external barrier. This method of stimulation imposes the least restrictions on ureteral dilatation. Dynamic interaction between urine and the ureteral wall during peristalsis was deliberated.

3.1 Flow Field and Wall Shear Stress. The numerical model was solved for two different peristaltic velocities to obtain the ureteral outlet flow rate. The flow rates were measured at the end of the ureteral peristaltic motion or propagation wave. The velocity data at the ureteral outlet was integrated to compute the ureteral outlet flow rate.

Figure 2 shows the pressure profile in the ureter during the peristaltic wave propagation. As can be seen from this figure, there is an adverse pressure gradient occurred following the contraction wave peak. This undesired pressure gradient caused the recirculation regions in that area. By analyzing the pressure profiles at different times during peristalsis, it was determined that the adverse pressure gradient near the contraction wave peak moved steadily along with the longitudinal propagation of the wave. This adverse pressure gradient created a backward flow in the opposite direction of the peristaltic wave motion that can be verified by the fluid velocity vectors (Fig. 3). In this plot, the length of the vectors shows the relative urine velocity magnitude. Figure 3 illustrates the backward flow development at the beginning of peristalsis when the contraction wave was entering the ureter. Only a short time after the wave start-up, the backward flow occurs around the wave so that it affects the ureteral entrance. The physical reason for this event is the effect of the inertial forces suddenly applied to urine flow owing to the wall stimulation. As the wave propagated toward the ureter, a chronic back flow developed completely, which moved downstream along with the contraction wave propagation (Fig. 3(c)), and the amounts of back flow at the ureteral inlet gradually diminished; so, ureteropelvic reflux is more probable to be present at the beginning of the wall peristaltic motion.

The results showed that the pressure gradient magnitude along the ureteral longitudinal direction on its wall, symmetry line was the maximum value around the peak of the propagating contraction wave, and these values decreased as a result of the wave dissemination in the longitudinal direction to the bladder, as is illustrated in Fig. 4.

The results also indicated that the wall shear stress near the peak of the moving contraction wave was the maximum. This stress decreased gradually by the peristaltic wave propagation toward the bladder (Fig. 5).

\subsection{Effect of Different Parameters on the Ureteral Outlet} Flow Rate

3.2.1 The Ureteral Wall Compliance. To understand the effect of the ureteral wall compliance on the urine flow rate in the nonperistaltic mechanism, two numerical experiments were performed on the finite element model. In these experiments, Young's modulus of $5 \mathrm{kPa}$ and $10 \mathrm{kPa}$ were utilized, which are in the physiological range of human ureteral elasticity [20]. As predicted, ureteral wall dilatation with a wall Young's modulus of 5 $\mathrm{kPa}$ was greater than that of the $10 \mathrm{kPa}$; so, there was an increase in the ureteral internal diameter, resulting from the imposed pressure (the ureteral average pressure was supposed to be $130 \mathrm{~Pa}$ ). With the assumption of $h$ being constant, the decrease in ureteral wall elasticity results in higher dilatation of the ureter, and therefore, fluid volume transported by the peristaltic wave and consequently the ureteral outlet flow rate are expected to increase. This expectation was completely verified by all our numerical results. For instance, the difference between the ureteral diametrical expansions in two cases with different wall Young's modulus can be seen from the ureteral outlet velocity magnitude profile in Fig. 6 and can be related to the different corresponding flow rate magnitudes. From this figure, ureteral dilatation difference at the radius of $3.5 \mathrm{~mm}$ is about $0.13 \mathrm{~mm}$. The results indicate that the
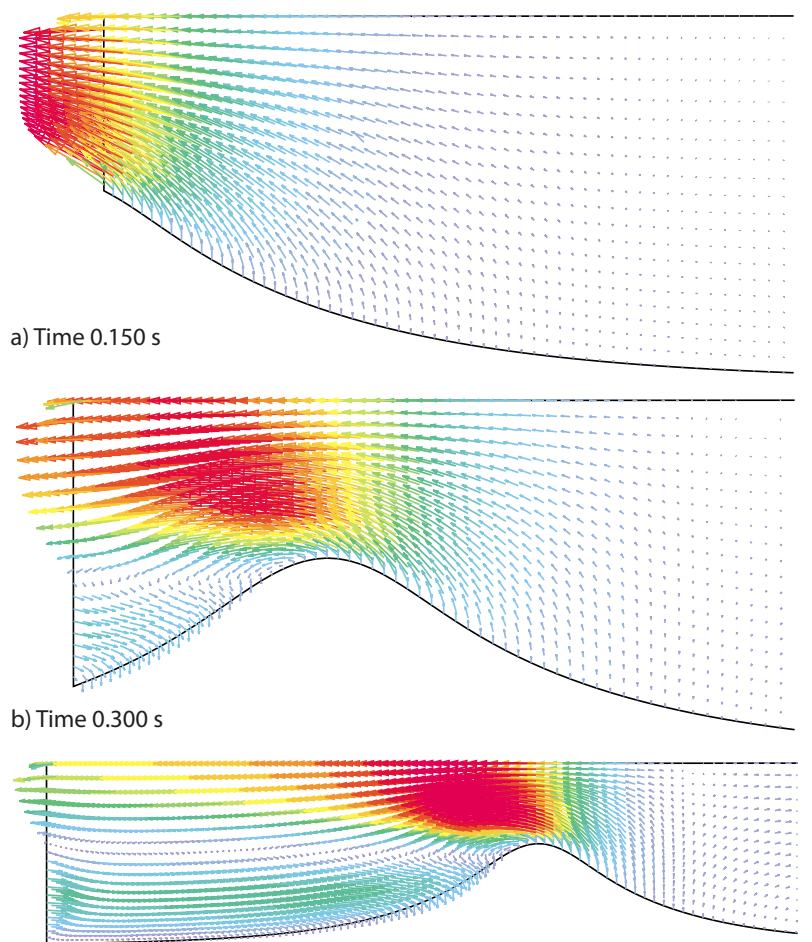

c) Time $0.650 \mathrm{~s}$

Fig. 3 Urine velocity vector plot showing ureteral back flow development following the contraction wave at the beginning of peristalsis. In this plot, time is considered from the beginning of peristalsis and the length of the vectors shows the relative velocity magnitude. (a) The contraction wave is entering into the ureter, the urine flow field varies slightly; (b) A moderate back flow is developing at the ureteral entrance; (c) A chronic back flow has been developed completely, which moves along with the contraction wave propagation downstream. 


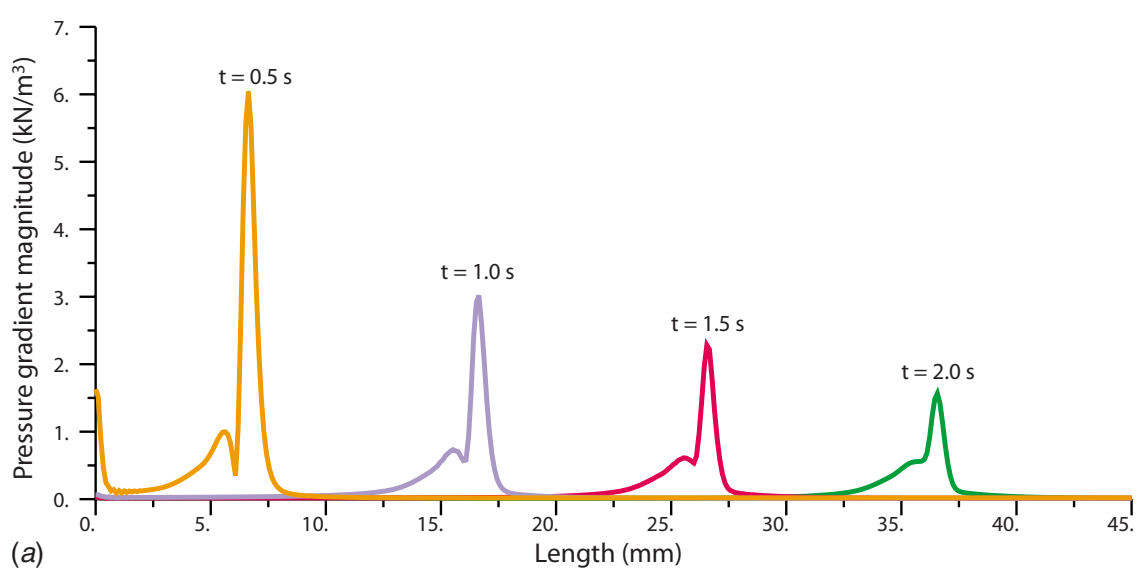

(a)

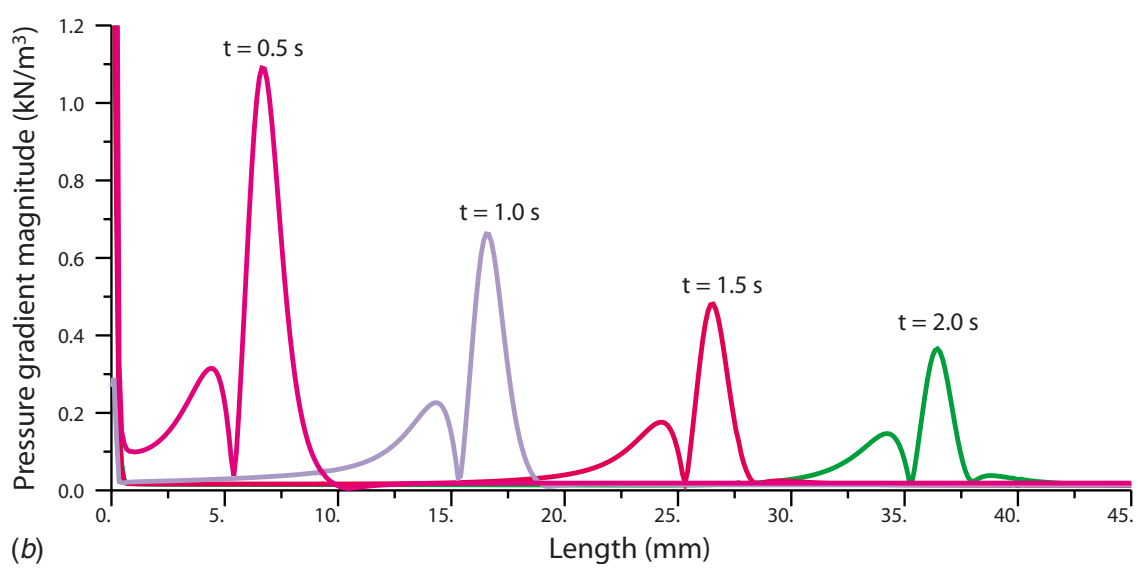

Fig. 4 Pressure gradient magnitude (a) along the ureteral wall and (b) along the ureteral symmetry line; the results are related to the case of $E=5 \mathrm{kPa}, \mathrm{V}$ $=2 \mathrm{~cm} / \mathrm{s}, \mathrm{h}=1.4 \mathrm{~mm}$, and $\Delta \mathrm{p}=0.3 \mathrm{~Pa}$. Times shown on the figure were measured from the beginning of peristalsis.

increase of ureteral wall compliance (decrease in ureteral wall Young's modulus) accordingly enhances the urine transportation capacity in the ureter.

In order to determine how much the wall compliance affects the ureteral outlet flow rate in the nonperistaltic and peristaltic mechanisms of urine transportation, we increased the wall elasticity up to $1 \mathrm{GPa}$, which minimizes the effect of wall compliance on flow and preserves the dynamic wall configuration like the other FSI models undergoing peristalsis that is needed for a true comparison between the models. The results shown in Fig. 6 illustrate the noticeable effect of wall compliance on urine flow in the nonperistaltic mechanism, and the results related to peristaltic mechanism will be presented in Sec. 3.2.4.

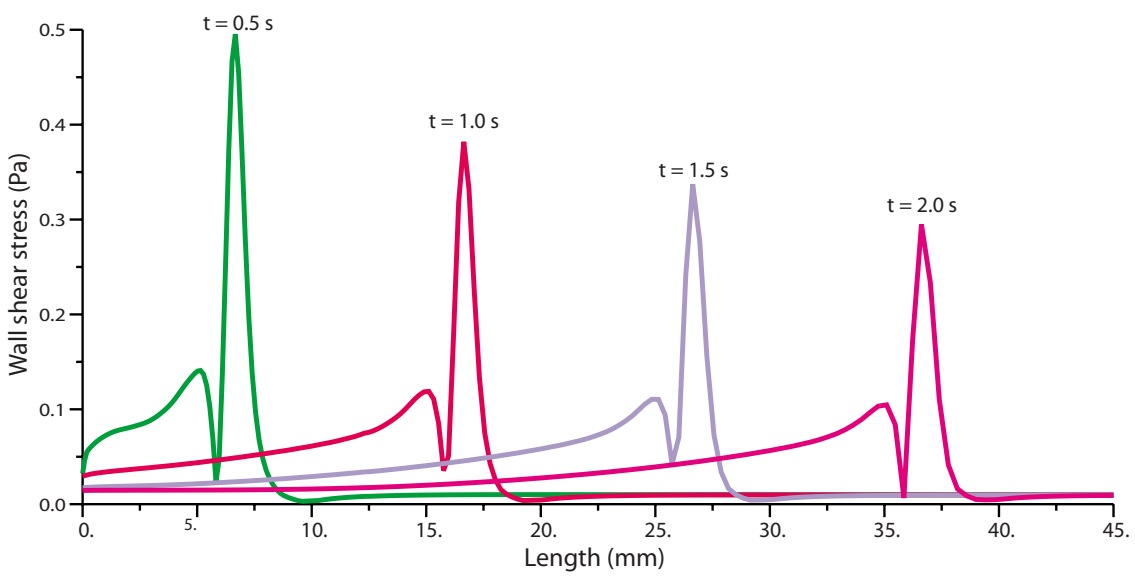

Fig. 5 Shear stress on the ureteral wall in the case of $E=5 \mathrm{kPa}, V=2 \mathrm{~cm} / \mathrm{s}, \mathrm{h}$ $=1.4 \mathrm{~mm}$, and $\Delta p=0.3 \mathrm{~Pa}$. Times shown on the figure were measured from the beginning of peristalsis. This pattern of shear stress reduction was observed in all the numerical experiments. 


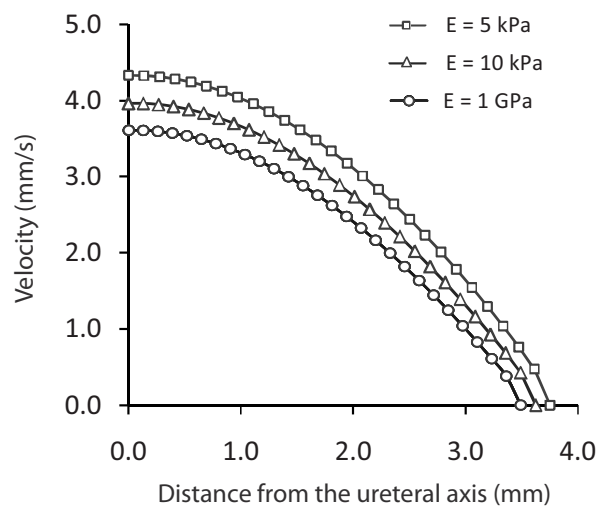

Fig. 6 Urine velocity magnitude versus the distance from the ureteral axis at the ureteral outlet in the case of nonperistaltic flow for two numerical experiments with $\Delta p=0.3 \mathrm{~Pa}$ and different Young's modulus of $E=5 \mathrm{kPa}, \mathrm{E}=10 \mathrm{kPa}$, and $\mathrm{E}=1 \mathrm{GPa}$.

3.2.2 The Peristaltic Wave Velocity. Figure 7 shows the urine velocity profile at the ureteral outlet for two different cases in which only the peristaltic wave velocity varies from $1 \mathrm{~cm} / \mathrm{s}$ to 2 $\mathrm{cm} / \mathrm{s}$ at $\mathrm{t}=12 \mathrm{~s}$ (peristaltic wave starting time) and at the end of the wall peristaltic activity in each model. Figure 7 also illustrates the condition in which the contraction wave moves with a low velocity magnitude. This motion does not have a considerable effect on the urine velocity magnitude; therefore, in these cases, the peristaltic mechanism does not have a noticeable effect on the ureteral outlet flow rate. Thus, the peristaltic efficiency is negligible. However, a contraction wave with a higher velocity (more than $1 \mathrm{~cm} / \mathrm{s}$ ) has a significant effect on the ureteral outlet flow rate.

3.2.3 Ureteral Outlet and Inlet Pressure Difference. In order to understand the effect of the peristaltic wave motion on the urine flow rate (peristaltic efficiency), one method is varying the ureteral inlet and outlet pressures and detecting the urine flow rate in the ureter, applying wall peristaltic motion. This situation was modeled with the assumption of three pressure differences $(+0.3$, 0 , and $-0.05 \mathrm{~Pa}$ ) between the ureteral inlet and outlet (the assumed pressure magnitudes that were applied to the ureteral inlet and outlet in different models are seen in Table 1). The effect of the pressure difference between the ureteral inlet and outlet on the urine flow rate at the ureteral outlet for different maximum heights

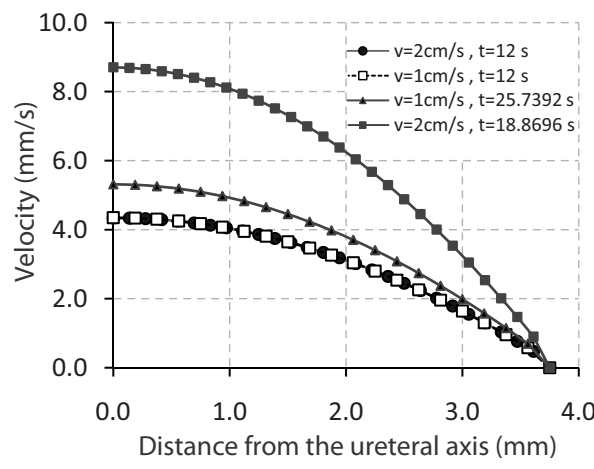

Fig. 7 Urine velocity magnitude versus the distance from the ureteral axis at the ureteral outlet for two numerical experiments with different peristaltic velocities of $V=1 \mathrm{~cm} / \mathrm{s}$ and $V$ $=2 \mathrm{~cm} / \mathrm{s}$. The curves shown in this figure relate to nonperistaltic flow at $t=12 \mathrm{~s}$ (the peristaltic wave beginning moment) and to the end of the wall peristaltic activity when the wave reaches the ureteral outlet. The effect of peristalsis on urine velocity can be seen from the figure obviously. The related parameters are $E=5 \mathrm{kPa}, \mathrm{h}=1.68 \mathrm{~mm}$, and $\Delta \mathrm{p}=0.3 \mathrm{~Pa}$.

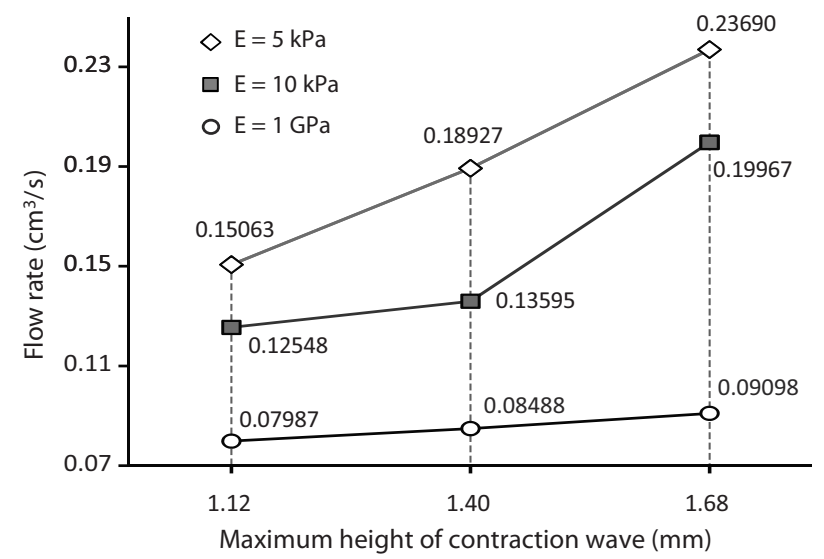

Fig. 8 Urine flow rate versus the maximum height of the contraction wave for three numerical experiments with different Young's modulus of $E=5 \mathrm{kPa}, \mathrm{E}=10 \mathrm{kPa}$, and $\mathrm{E}=1 \mathrm{GPa}$. The related parameters are $\mathrm{V}=2 \mathrm{~cm} / \mathrm{s}$ and $\Delta \mathrm{p}=0.3 \mathrm{~Pa}$.

of contraction waves is given in Table 1. For a better comparison, the ureteral outlet flow rate was calculated considering peristalsis and without wall peristaltic activity (at $\mathrm{t}=12 \mathrm{~s}$ before the peristalsis start-up) and can be seen in Table 1 and Fig. 8. Our results show that the urine flow rate increased by the peristaltic wave propagation up to three times the nonperistaltic flow (which can be simply calculated from the values given in Table 1 and Fig. 8).

3.2.4 The Maximum Height of Contraction Wave. Figure 8 illustrates the relations among the ureteral flow rate, wall elasticity, and the maximum height of the contraction wave as a result of the ureteral peristaltic activity. As illustrated in Fig. 8, the ureteral outlet flow rate and hence the peristaltic efficiency increased by increasing the maximum height of the contraction wave. This occurred owing to a greater force being applied to the running fluid because of a greater constriction in the ureteral lumen as a result of wall stimulation.

The bilateral effects of the wall elasticity and the maximum height of the contraction wave on the flow rate are also illustrated in Fig. 8. Wall rigidity effects have also been considered in this study assuming $\mathrm{E}=1 \mathrm{GPa}$ in order to determine how much wall compliance affects the urine flow via peristalsis, which can be seen in this figure. The lower magnitudes of flow rate in this figure relate to $\mathrm{E}=1 \mathrm{GPa}$ in which the flow rate increases up to $13.9 \%$ by increasing $\mathrm{h}$ from 1.12 to 1.68 , while in $\mathrm{E}=10 \mathrm{kPa}$ and $\mathrm{E}$ $=5 \mathrm{kPa}$, the flow rate increases up to $59.1 \%$ and $57.3 \%$, respectively. Flow increases between $57.1 \%$ (the difference between $\mathrm{E}$ $=1 \mathrm{GPa}$ and $\mathrm{E}=10 \mathrm{kPa}$ in $\mathrm{h}=1.12$ ) up to $160 \%$ (the difference between $\mathrm{E}=1 \mathrm{GPa}$ and $\mathrm{E}=5 \mathrm{kPa}$ in $\mathrm{h}=1.68$ ). As a consequence, the reduction of wall elasticity causes a greater flow rate in different values of the maximum height of the contraction wave but not in a linear pattern.

3.2.5 Effect of the Simultaneous Number of Contraction Waves. One of the major factors analyzed in this paper is the effect of a number of simultaneous wave contractions in the ureter and its effect on the ureteral outlet flow rate. In order to simulate this situation, the second rigid surface, which is the inducer of the second contraction wave, was moved from the same coordinate by the same velocity along the ureter with a $5 \mathrm{~s}$ delay. The results of the flow rate taken from the ureteral outlet are presented in Fig. 9. In Fig. 9, the data of one contraction wave is also presented for comparison. The following items have been considered in each solution: (1) The moment of peristalsis start-up ( $t=12 \mathrm{~s})$ and (2) the overall solution time that lasts during $t=0$ up to $t=26 \mathrm{~s}$ including the time interval of nonperistaltic phase in all of the models as well as the time interval in the model of one contraction wave, and at last, the time interval in the models of two simulta- 


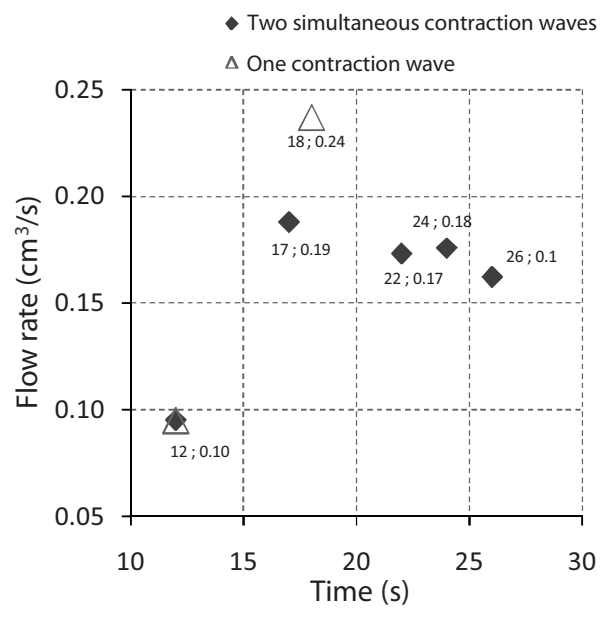

Fig. 9 Urine flow rate at the ureteral outlet during peristalsis. This figure shows the effect of the number of contraction waves on the amount of urine transported in the ureter. The moment of peristalsis start-up is at $t=12 \mathrm{~s}$, and the overall solution time lasts during $t=0$ up to $t=26 s$ including the time interval of nonperistaltic phase in all of the models as well as the time interval in the model of one contraction wave, and at last, the time interval in the models of two simultaneous contraction waves during peristalsis with a delay of $5 \mathrm{~s}$ between the two successive contraction waves. The related parameters are $\mathrm{E}=5 \mathrm{kPa}, \mathrm{V}=2 \mathrm{~cm} / \mathrm{s}, \mathrm{h}=1.68 \mathrm{~mm}$, and $\Delta \mathrm{p}=0.3 \mathrm{~Pa}$.

neous contraction waves during peristalsis with a delay of $5 \mathrm{~s}$ between the two successive contraction waves. It is obvious from Fig. 9 that a greater volume of urine per unit time is transported in the ureter in the model having one peristaltic wave than in the models having two simultaneous propagating contraction waves. Also, by the further propagation of the second wave in the ureter, the outlet flow rate decreases more.

\section{Discussion}

In general, four types of flow regimes were reported to occur in the ureter through peristalsis; they are isolated bolus, boluses in contact, leaky bolus, and open-tube flow $[15,16,33]$. This study is limited to the open-tube flow regime, which has the highest flow rate among the aforementioned regimes. Also, the simulation is restricted to the condition in which the pressure difference between the renal pelvis and the bladder is insignificant.

After a thorough analysis of the flow field results (Fig. 3), it was concluded that ureteropelvic junction (UPJ) dysfunction (as modeled in this paper) results in the retrograde flow of urine even when there is a slow (not accelerated) start-up of the contraction wave. The reduction in the pressure gradient magnitude during the peristaltic wave propagation (Fig. 4) is due to the spontaneous acceleration of the fluid around the contraction wave and the subsequent immediate phase of urine deceleration, which is a result of the wave longitudinal propagation.

The ureteral wall stress results (Fig. 5) indicated that the maximum values of shear stress around the peak of the wave decreased by the wave longitudinal propagation; so, it can be concluded that the proximal portion of the ureter, which is near the kidney, is prone to a higher shear stress during the ureteral peristaltic mechanism. High values of shear stress at the beginning of the ureteral peristaltic activity is due to the spontaneous acceleration of the urine, which results in a large relative velocity difference between the urine near the wall (at the position of the contraction wave) and the whole urine in the ureter. This noticeable difference reduces gradually by the wave longitudinal propagation.

For the nonperistaltic mechanism of urine transport via the ureter (Fig. 6), considering the fact that the ureteral outlet flow rate is proportional to its diameter, we can manipulate the effect of the diametrical parameter by variation of two factors, (1) at the same wall compliance, assuming different diametrical values, and (2) at the same geometrical condition (the equivalent diameters), assuming different compliance for the ureteral wall. In the latter situation, an increase in wall compliance (reduction of Young's modulus) increases the ureteral wall dilatation and therefore results in flow rate increase.

For the effect of the peristaltic wave velocity on ureteral outlet flow rate (Fig. 7), it can be concluded that on every occasion that the velocity of the contraction wave is a low magnitude comparing to the time scale of fluid motion, the condition is so that the fluid does not sense the contraction wave movement and the contraction wave plays the role of a stenosis in the ureter, which leads to the increase of the ureteral resistance that results in the decrease of ureteral outlet flow rate.

The obtained results regarding peristaltic efficiency are in good agreement with theory as well as previously published studies $[29,34]$. In particular, the results pertaining to the maximum height of contraction wave (Fig. 8) are in accordance with findings of Carew and Pedley [34]. Making use of a mathematical simulation, they demonstrated that the constriction of the ureteral lumen during peristalsis has a major effect on peristaltic efficiency. They proved that the ureteral peristaltic pumping is most efficient in the case of a ureter, which lumen can theoretically be pressed shut. Walker and Shelly [29], using a variational method for optimizing the shape of a peristaltic wave, reported that peristaltic flux increases by increasing the amount of occlusion in a 2D channel. These findings were confirmed by our results in Fig. 8. They also showed that flux increases linearly by the occlusion. This result does not agree with our numerical results presented in Fig. 8. The contradiction seems to be due to adding the wall compliance in our models. It is illustrated in Fig. 8 that as the height of the contraction wave becomes smaller, the effect of wall elasticity on the urine flow rate decreases comparing $\mathrm{E}=5 \mathrm{kPa}$ with $\mathrm{E}=10 \mathrm{kPa}$. The reason is that the situation is approaching to the completely open-tube condition, where the moving obstruction in the ureter is negligible and therefore, the peristaltic efficiency is near zero. By increasing the maximum height of the contraction wave, the effect of wall elasticity on the urine flow rate increases although in cases of nearly complete occlusion of the ureteral lumen during peristalsis, the effect of wall elasticity on the urine flow rate (the difference between the magnitudes of flow rates in Fig. 8) decreases because of the dominant effect of fluid high pressure gradients around the peak of the propagating contraction wave. The noticeable effect of wall compliance on urine flow through peristalsis can be concluded comparing the magnitudes of flow rate in $\mathrm{E}=1 \mathrm{GPa}$ (which minimizes the effect of wall compliance on flow) with $\mathrm{E}=5 \mathrm{kPa}$ and $\mathrm{E}=10 \mathrm{kPa}$ (which are in the physiological range of ureteral wall elasticity).

The simultaneous contraction waves and their effect on the ureter of pigs and humans have been investigated by biological experimentalists $[2,15,16]$. As it can be understood from Fig. 9 and because of the fact that contraction waves locally restrict the flow around the peak of the wave (the lumen area decreases), the reason for flow rate reduction in the models with two simultaneous contraction waves is the greater pressure drop along the ureter. This greater pressure drop is caused by the presence of two moving constrictions compared with one moving constriction in the model with one contraction wave. In other words, despite enhancement of the urine flow propelling force by two contraction waves, the adverse effect of a pressure drop along the ureter is dominant, and thus an overall flow rate reduction occurs.

The results revealed a significant role of fluid-structure interaction on flow and stress analysis within a uniform compliant tube undergoing peristalsis. Similar to other models that simulate a system as sophisticated as a living organism, we made some simplifications in our approach. These simplifications were mostly in geometry and material properties. Mean physiological values from Yin and Fung [20] have been used for ureteral wall material prop- 
erties. The ureteral wall was assumed to be straight, linearly elastic, homogeneous, and isotropic. Clearly, mammalian ureters exhibit wide variations in terms of shape, size, material properties, and loading conditions. Carew and Pedley [34] considered the viscoelastic properties of ureteral wall based on the reasonable assumption of the thin shell theory. However, their studies, which models periodic activation waves (a nonrealistic biological assumption because of time independency of the equations in the wave frame) in an infinite tube, did not account for cell-to-cell stimulation of ureteral wall that describes the physiological conditions of peristaltic wave longitudinal propagation.

In addition to aforementioned complications regarding the geometry and material properties, there were also some difficulties pertaining to stability of the solutions of FSI equations. These difficulties mainly relate to the loading conditions of the ureteral wall in the FSI model. Since in this approach the interactions between urine and the ureter are also taken into account, the complications in each of the fluid and solid models are even more pronounced. With this in mind, it is essential to reduce uncertainties in the fluid and solid models prior to introduction of wall motion to the model that was sensitively satisfied in this study.

We assumed a fixed-velocity for the movement of the wall stimulator, i.e., the average velocity of peristalsis $[22,28]$, which allows for peristaltic wave propagation through the ureter. It should be noted that in this paper, we did not consider the physiological conditions of ureter undergoing higher luminal pressure level, which varies between $20 \mathrm{mmHg}$ and $40 \mathrm{mmHg}$ in healthy ureters [28], due to computational restrictions although by applying pressure loadings to the ureteral inlet and outlet, we assumed approximately the true constant level of pressure in the ureter that is between $1 \mathrm{mmHg}$ and $6 \mathrm{mmHg}$ [28]. In the physiological function of ureteral peristalsis, the contraction force generated by the smooth muscle depends on the luminal pressure [1]. Therefore, the prescribed radial fixation of the mechanical stimulator (rigid surface) during peristalsis in our model is another limitation that makes the maximum constriction independent of luminal pressure. The assumed axisymmetric geometry of the ureter model with uniform thickness is highly idealized and was performed to simplify the case so that the solution will be numerically tractable. More complicated shapes are required to model realistic ureteral dilatation due to the stellate form of the ureteral luminal area [8]. Such shapes will require the spatial finite element discretizations. Nonetheless, we should emphasize that the goal of this work was not to model an isolated urine bolus movement in the ureter but rather to develop a mathematical framework suitable for simulating the ureteral peristalsis. The use of mechanical stimulation to induce peristalsis in the ureteral wall is novel and has not been previously addressed in the literature.

4.1 Future Studies. There are still many unsolved problems in this field to which the introduced approach can be applied. For example, the complicated nature of reflux and the meaning of several diagnostic indicators are not well understood. The direct interaction between the two parts of the urinary system (kidney and bladder) has scarcely received any theoretical contemplation. Furthermore, the wall activation by a pacemaker that induces peristalsis and the detailed process of the elastic deformation in the ureteral muscle, which is highly nonlinear, has not been fully explained yet.

The introduced model needs some improvements in future works to get closer to reality. For highly deformable ureteral wall, nonlinear hyperelastic and viscoelastic models might represent ureteral wall more realistically. The aspects of initial stress and strain have not been incorporated in our proposed models yet although their effects are not expected to invalidate the results of this study. The assumption of a time-dependent peristaltic velocity based on in vivo data also results in a more realistic simulation and should be considered in future studies.

The modeling introduced in this paper may also be used to study the effect of non-Newtonian fluids on peristaltic pumping, which was investigated by Teran et al. [30], with 2D models using immersed boundary method. Moreover, the nonreturn action of the ureterovesical junction (UVJ), which prevents the back flow from the bladder into the ureter, is an important phenomenon that can be simulated and better understood by our presented approach. Patient-specific modeling of ureteral mechanics based on geometric data using dynamic magnetic resonance imaging (MRI) or computed tomography (CT) imaging will be valuable to identify crucial disease conditions.

\section{Conclusions}

In this paper, a numerical simulation with FSI for urine transport in the ureter was performed. This simulation introduces a new approach to evaluate more accurately the peristaltic mechanism in the ureter. The effect of physiological factors that influence the efficiency of the peristaltic process was investigated. These factors include the pressure difference among the kidney and the bladder, the ureteral wall compliance, the peristaltic wave velocity, the maximum height of the contraction wave, and the number of contraction waves that affect the peristaltic efficiency, ureteral wall shear stress, and the urine flow field. Our results indicated that there is a high possibility of occurrence of reflux at the beginning of peristalsis in the ureter. Moreover, the peristaltic efficiency is highly dependent on the ureteral wall compliance, and therefore, fluid-structure interaction plays an important role in analyzing the ureteral fluid dynamics.

\section{References}

[1] Fung, Y. C., 1971, "Peristaltic Pumping: A Bioengineering Model," Urody namics: Hydrodynamics of the Ureter and Renal Pelvis, S. Boyarsky, G. W. Gottschalk, E. A. Tanagho, and P. D. Zimskind, eds., Academic, New York, pp. 189-198.

[2] Griffiths, D. J., Constantinou, E. C., Mortensen, J., and Djurhuus, J. C., 1987, "Dynamics of the Upper Urinary Tract: II. The Effect of Variations of Peristaltic Frequency and Bladder Pressure on Pyeloureteral Pressure/Flow Relations," Phys. Med. Biol., 32(7), pp. 823-833.

[3] Bykova, A. A., and Regirer, S. A., 2005, "Mathematical Models in Urinary System Mechanics,” J. Fluid Mech., 40(1), pp. 221-226.

[4] Uehara, Y., and Burnstock, G., 1970, "Demonstration of "Gap Junctions" Between Smooth Muscle Cells," J. Cell Biol., 44, pp. 215-217.

[5] Eccles, M. R., 1998, "The Role of PAX2 in Normal and Abnormal Development of the Urinary Tract," Pediatr. Nephrol., 12, pp. 712-720.

[6] Saeki, H., Morita, T., Nishimoto, T., Kondo, S., and Tsuchida, S., 1985 , "Changes in the Ureteral Peristaltic Rate and the Bolus Volume in Gradual and Rapid Urinary Flow Increase,” Tohoku J. Exp. Med., 146, pp. 273-275.

[7] Zelenko, N., and Coll, D., 2004, "Normal Ureter Size on Unenhanced Helical CT," AJR, Am. J. Roentgenol., 182, pp. 1039-1041.

[8] Woodburne, R. T., and Lapides, J., 1972, "The Ureteral Lumen During Peristalsis," Am. J. Anat., 133(3), pp. 255-258.

[9] Kontani, H., Ginkawa, M., and Sakai, T., 1993, "A Simple Method for Measurement of Ureteric Peristaltic Function In Vivo and the Effects of Drugs Acting on Ion Channels Applied From the Ureter Lumen in Anesthetized Rats," Jpn. J. Pharmacol., 62, pp. 331-338.

[10] Jørgensen, T. M., 1986, "Pathogenetic Factors in Vesicoureteral Reflux," Neurourol Urodyn, 5, pp. 153-183.

[11] Liu, J. X., Park, Y. C., Mah, S. Y., Smith, D., Woodard, S., McGuire, E. J., Wein, A., Levin, R., Miller, L. F., and Elbadawi, A., 1991, "Ureteral Perfusion in Normal and Chronically Obstructed Feline Models," Korean J. Urol., 32(6), pp. 980-985.

[12] Mahoney, Z. X., Sammut, B., Xavier, R. J., Cunningham, J., Go, G., Brim, K. L., Stappenbeck, T. S., Miner, J. H., and Swat, W., 2006, "Discs-Large Homolog 1 Regulates Smooth Muscle Orientation in the Mouse Ureter," Proc. Natl. Acad. Sci. U.S.A., 103(52), pp. 19872-19877.

[13] Aragona, F., Artibani, W., de Caro, R., Pizzarella, M., and Passerini, G., 1988, "The Morphological Basis of Ureteral Peristalsis," Int. Urol. Nephrol., 20(3), pp. 239-250.

[14] Melchior, H., 1975, "Urodynamics," Urol. Res., 3, pp. 51-54.

[15] Ohlson, L., 1989, "Morphological Dynamics of Ureteral Transport I. Shape and Volume of Constituent Urine Fractions," Am. J. Physiol., 256, pp. R19R28.

[16] Ohlson, L., 1989, "Morphological Dynamics of Ureteral Transport II. Peristaltic Patterns in Relation to Flow Rate," Am. J. Physiol. 256, pp. R29-R34.

[17] Fung, Y. C., 1993, Biomechanics: Mechanical Properties of Living Tissues, Springer-Verlag, New York.

[18] Hansen, I., and Gregersen, H., 1999, "Morphometry and Residual Strain in Porcine Ureter," Scand. J. Urol. Nephrol., 33, pp. 10-16.

[19] Knudsen, L., Gregersen, H., Eika, B., and Frøkiær, J., 1994, "Elastic Wall Properties and Collagen Content in the Ureter: An Experimental Study in Pigs," Neurourol Urodyn, 13, pp. 597-606. 
[20] Yin, F. C. P., and Fung, Y. C., 1971, "Mechanical Properties of Isolated Mammalian Ureteral Segments," Am. J. Physiol., 221(5), pp. 1484-1493.

[21] Gregersen, H., and Kassab, G., 1996, "Biomechanics of the Gastro-Intestinal Tract," Neurogastroenterol Motil, 8, pp. 277-297.

[22] Gintz, D., Elmabsout, B., and Renaudeaux, J. P., 1999, "Modelling of the Urine Flow in the Human Ureter," Acad. Sci., Paris, C. R., 327, pp. 12651268.

[23] Gintz, D., Elmabsout, B., and Renaudeaux, J. P., 2001, "Modelling of the Human Ureteral Bolus," Acad. Sci., Paris, C. R., 329, pp. 303-306.

[24] Griffiths, D. J., and Notschaele, C., 1983, "The Mechanics of Urine Transport in the Upper Urinary Tract: The Dynamics of the Isolated Bolus," Neurouro Urodyn, 2, pp. 155-166.

[25] Vogel, A., Elmabsout, B., and Gintz, D., 2004, "Modelling of Urine Flow in a Ureteral Bolus," C. R. Mec., 332, pp. 737-742.

[26] Eytan, O., Jaffa, A. J., and Elad, D., 2001, "Peristaltic Flow in a Tapered Channel: Application to Embryo Transport Within the Uterine Cavity," Med. Eng. Phys., 23, pp. 475-484.

[27] Misra, J. C., and Pandey, S. K., 2001, "A Mathematical Model for Oesophageal Swallowing of a Food-Bolus," Math. Comput. Modell., 33, pp. $997-$
1009

[28] Jiménez-Lozano, J., 2009, "Peristaltic Flow With Application to Ureteral Biomechanics," Ph.D. thesis, Notre Dame University, Indiana.

[29] Walker, S. W., and Shelley, M. J., 2010, Shape Optimization of Peristaltic Pumping," J. Comput. Phys., 229, pp. 1260-1291.

[30] Teran, J., Fauci, L., and Shelley, M. J., 2008, "Peristaltic Pumping and Irreversibility of a Stokesian Viscoelastic Fluid," Phys. Fluids, 20, p. 073101-1073101-11.

[31] Vahidi, B., and Fatouraee, N., 2007, "Mathematical Modeling of the Uretera Peristaltic Flow With Fluid Structure Interaction,” J. Biomech., 40, p. S223.

[32] Bykova, A. A., and Regirer, S. A., 1999, "Simple Model of Peristalsis in a Myogenically-Active Tube," Euromech Colloquium 389, Book of Abstracts, Graz, Austria, pp. 68-69.

[33] Griffiths, D. J., 1987, "Dynamics of the Upper Urinary Tract: I. Peristaltic Flow Through a Distensible Tube of Limited Length," Phys. Med. Biol. 32(7), pp. 813-822.

[34] Carew, E. O., and Pedley, T. J., 1997, "An Active Membrane Model for Peristaltic Pumping. Pt 1. Periodic Activation Waves in an Infinite Tube," ASME J. Biomech. Eng., 119(1), pp. 66-76. 